\title{
ON CLOSED GEODESICS IN THE LEAF SPACE OF SINGULAR RIEMANNIAN FOLIATIONS
}

\author{
MARCOS M. ALEXANDRINO \\ Instituto de Matemática e Estatística Universidade de São Paulo, Rua do Matão \\ 1010,05508 090 São Paulo, Brazil \\ e-mail:marcosmalex@yahoo.de,malex@ime.usp.br \\ and MIGUEL ANGEL JAVALOYES \\ Departamento de Geometría y Topología. Facultad de Ciencias, Universidad de Granada. \\ Campus Fuentenueva s/n, 18071 Granada, Spain \\ e-mail:majava@ugr.es,ma.javaloyes@gmail.com
}

(Received 21 April 2010; revised 11 August 2010; accepted 21 September 2010; first published online 10 March 2011)

\begin{abstract}
In this paper we prove the existence of closed geodesics in the leaf space of some classes of singular Riemannian foliations (s.r.f.), namely s.r.fs. that admit sections or have no horizontal conjugate points. We also investigate the shortening process with respect to Riemannian foliations.
\end{abstract}

2010 Mathematics Subject Classification. Primary 53C12, Secondary 57R30.

1. Introduction. Guruprasad and Haefliger [11, Theorem 5.1.1, Remark 5.1.2] proved the existence of closed geodesics in a Riemannian compact orbifold $Q$ (recall Definition 2.4 and Definition 2.15) in the following cases:

(1) $Q$ is not developable (not good orbifold).

(2) $Q$ is a good orbifold $\Sigma / W$ and $W$ has an element of infinite order or is finite.

The aim of this paper is two-fold. The first one is to prove the existence of closed geodesics in the leaf space of some classes of singular Riemannian foliations (s.r.f.). We start by recalling some concepts of orbifold theory and by giving an alternative proof of item (2) of Guruprasad and Haefliger's theorem (see Proposition 2.16). Then using the result of Guruprasad and Haefliger [11], Hosaka [12] and Lytchak [14], conclude the existence of closed geodesics in $M / \mathcal{F}$ when $\mathcal{F}$ is an s.r.f. with closed and embedded leaves on a simply connected complete Riemannian manifold $M$, and $M / \mathcal{F}$ is a compact orbifold (see Theorem 2.20). In particular, we show the existence of a closed geodesic of the orbifold $M / \mathcal{F}$ when $\mathcal{F}$ admits sections (e.g. the partition of a polar action by orbits) or $\mathcal{F}$ has no horizontal conjugate points (e.g. the partition by orbits of a variationally complete action), and $M / \mathcal{F}$ is compact and $M$ is simply connected (see Corollary 2.22).

The second aim of this paper is to construct the shortening process with respect to Riemannian foliations (see Section 3). This provides an algorithm to find closed geodesics in some special Riemannian orbifolds.

The first author was supported by CNPq and partially supported by FAPESP. The second author was partially supported by Regional J. Andalucía Grant P09-FQM-4496, by MICINN project MTM2009-10418 and Fundación Séneca project 04540/GERM/06. 
This paper is organised as follows. In Section 2 we present and prove the main results of this paper, i.e. Theorem 2.20 and Corollary 2.22. In Section 3 we present the shortening process with respect to Riemannian foliations and infer some results (e.g. Proposition 3.9 and Corollary 3.14).

2. Orbifolds and Riemannian foliations. In this section, we recall some definitions, state our main results as Theorem 2.20 and Corollary 2.22 and give concise proofs for them.

We start by recalling some facts about orbifolds. More details can be found in Salem [18, Appendix D], Guruprasad and Haefliger [11] or Moerdijk and Mrčun [17].

DEFINITION 2.1 (Riemannian pseudogroup). Let $\Sigma$ be a Riemannian manifold, not necessarily connected. A pseudogroup $W$ of isometries of $\Sigma$ is a collection of local isometries $w: U \rightarrow V$, where $U$ and $V$ are open subsets of $\Sigma$ such that

(1) If $w \in W$, then $w^{-1} \in W$.

(2) If $w: U \rightarrow V$ and $\tilde{w}: \tilde{U} \rightarrow \tilde{V}$ belong to $W$, then $\tilde{w} \circ w: w^{-1}(\tilde{U} \cap V) \rightarrow$ $\tilde{w}(\tilde{U} \cap V)) \subset \tilde{V}$ also belongs to $W$, if $V \cap \tilde{U} \neq \varnothing$.

(3) If $w: U \rightarrow V$ belongs to $W$, then its restriction to each open subset $\tilde{U} \subset U$ also belongs to $W$.

(4) If $w: U \rightarrow V$ is an isometry between open subsets of $\Sigma$ that coincides in a neighbourhood of each point of $U$ with an element of $W$, then $w \in W$.

Definition 2.2. Let $A$ be a family of local isometries of $\Sigma$ containing the identity map of $\Sigma$. The pseudogroup obtained by taking the inverses of the elements of $A$, the restrictions of elements of $A$ to open subsets, as well as their compositions and their unions, is called the pseudogroup generated by $A$.

An important example of a Riemannian pseudogroup is the holonomy pseudogroup of a Riemannian foliation, whose definition we now recall.

DEFINITION 2.3. Let $\mathcal{F}$ be a foliation of codimension $k$ on a Riemannian manifold $(M, \mathrm{~g})$. Then $\mathcal{F}$ is a Riemannian foliation if it can be described by an open cover $\left\{U_{i}\right\}$ of $M$ with Riemannian submersions $f_{i}:\left(U_{i}, \mathrm{~g}\right) \rightarrow\left(\sigma_{i}, b\right)$ (where $\sigma_{i}$ is a submanifold of dimension $k$ ) such that there are isometries $w_{i, j}: f_{i}\left(U_{i} \cap U_{j}\right) \rightarrow f_{j}\left(U_{j} \cap U_{i}\right)$ with $f_{j}=w_{i, j} \circ f_{i}$. The elements $w_{i, j}$ acting on $\Sigma=\amalg \sigma_{i}$ generate a pseudogroup of isometries of $\Sigma$ called the holonomy pseudogroup of $\mathcal{F}$.

Definition 2.4 (Riemannian orbifold). One can define the $k$-dimensional Riemannian orbifold as an equivalence class of pseudogroups $W$ of isometries on a Riemannian manifold $\Sigma$ (dimension of $\Sigma$ is equal to $k$ ) verifying the following conditions:

(1) The space of orbits $|\Sigma / W|$ is Hausdorff.

(2) For each $x \in \Sigma$, there exists an open neighbourhood $U$ of $x$ in $\Sigma$ such that the restriction of $W$ to $U$ is generated by a finite group of isometries of $U$.

In addition, if $W$ is a discrete subgroup of isometries of $\Sigma$ whose action on $\Sigma$ is proper, then $\Sigma / W$ is said good (for definition and properties of proper actions, see, for example, Duistermaat and Kolk [9]).

REMARK 2.5. Let $\Sigma / W$ be a Riemannian good orbifold. Since the action $W \times$ $\Sigma \rightarrow \Sigma$ is proper, one can conclude that $W$ is a closed subgroup of isometries of $\Sigma$ with discrete topology and the isotropy group $W_{x}$ is finite for each $x \in \Sigma$. 
REMARK 2.6. An important example of a Riemannian orbifold is the space of leaves $M / \mathcal{F}$, where $M$ is a complete Riemannian manifold and $\mathcal{F}$ is a Riemannian foliation on $M$ with closed and embedded leaves. In fact $M / \mathcal{F}$ turns out to be isomorphic to $\Sigma / W$, where $\Sigma$ and $W$ were presented in Definition 2.3. This is proved in Molino [18, Proposition 3.7] when the leaves are compact. In order to prove the case where the leaves are closed and embedded, it suffices to generalise Lemma 3.7 in [18] (using e.g. Claim 1 of Proposition 2.18 [6]). The proof, mutatis mutandis, now follows Molino's proof [18].

REMARK 2.7. There exists a reciprocal result, namely each Riemannian orbifold $\Sigma / W$ is the space of leaves of a Riemannian foliation with compact leaves. In fact Moerdijk and Mrčun [17, Proposition 2.23] proved that if $U(E)$ is the unitary frame bundle of the complexification of the tangent bundle of $\Sigma$, then $U(E) / W$ admits a foliation $\mathcal{F}^{u}$ such that $\Sigma / W$ is the orbifold $(U(E) / W) / \mathcal{F}^{u}$. Using the Riemannian connection of $\Sigma$ one can induce a distribution in $U(E)$ and $U(E) / W$, and then find an appropriate transverse metric such that the plaques of $\mathcal{F}^{u}$ can be described by the local Riemannian submersions.

Given a pseudogroup, and in particular an orbifold, we can define a fundamental group as we now recall.

Definition 2.8. A $W$-loop with base point $x_{0} \in \Sigma$ is defined by

(1) a sequence $0=t_{0}<\cdots<t_{n}=1$,

(2) continuous paths $c_{i}:\left[t_{i-1}, t_{i}\right] \rightarrow \Sigma, 1 \leq i \leq n$,

(3) elements $w_{i} \in W$ defined in a neighbourhood of $c_{i}\left(t_{i}\right)$ for $1 \leq i \leq n$ such that $c_{1}(0)=w_{n} c_{n}(1)=x_{0}$ and $w_{i} c_{i}\left(t_{i}\right)=c_{i+1}\left(t_{i}\right)$, where $1 \leq i \leq n-1$.

A subdivision of a $W$-loop is obtained by adding new points to the interval $[0,1]$, by taking the restriction of the $c_{i}$ to these new intervals and $w=i d$ at the new points.

Definition 2.9. Two $W$-loops are equivalent if there exists a subdivision common to the loops represented by $\left(c_{i}, w_{i}\right)$ and $\left(\tilde{c}_{i}, \tilde{w}_{i}\right)$ and elements $g_{i} \in W$ defined in a neighbourhood of the path $c_{i}$ such that

(1) $g_{1}=i d, g_{i} \circ c_{i}=\tilde{c}_{i}, 1 \leq i \leq n$,

(2) $\tilde{w}_{i} \circ g_{i}$ and $g_{i+1} \circ w_{i}$ have the same germ at $c_{i}\left(t_{i}\right), 1 \leq i \leq n-1$,

(3) $\tilde{w}_{n} \circ g_{n}$ has the same germ at $c_{n}(1)$ as $w_{n}$.

Definition 2.10. A deformation of a $W$-loop represented by $\left(c_{i}, w_{i}\right)$ is given by continuous deformations $c_{i}(s, \cdot)$ of the paths $c_{i}=c_{i}(0, \cdot):\left[t_{i-1}, t_{i}\right] \rightarrow \Sigma$ such that $\left(c_{i}(s, \cdot), w_{i}\right)$ represents a $W$-loop.

Definition 2.11. Two $W$-loops are in the same homotopy class if one can be obtained from the other by a series of subdivisions, equivalences and deformations. The homotopy classes of $W$-loops based at $x_{0} \in \Sigma$ form a group $\pi_{1}\left(W, x_{0}\right)$ called the fundamental group of the pseudogroup $W$ at the point $x_{0}$.

REMARK 2.12. If the orbit space $\Sigma / W$ is connected, then there exists an isomorphism, defined up to conjugation, between $\pi_{1}(W, x)$ and $\pi_{1}(W, y)$ for $x, y$ in $\Sigma$. Thus, we will write just $\pi_{1}(W)$ when convenient.

DEFINITION 2.13. If $\Sigma / W$ is a connected orbifold, its fundamental group $\pi(\Sigma / W)$ is defined as the fundamental group $\pi_{1}(W)$ of the pseudogroup $W$. 
REMARK 2.14. The fundamental group of an orbifold $\Sigma / W$ is not the same as the fundamental group of the topological space $|\Sigma / W|$. One of the differences lies in item (2) of Definition 2.9. In order to understand this claim, consider $W$, the group generated by the reflection in the line $\{x=0\}$ in $\mathbb{R}^{2}$. We note that the line that joins $(-1,0)$ to $(1,0)$ is a non-trivial element of the fundamental group of the orbifold $\mathbb{R}^{2} / W$. If we would remove the words have the same germ at in item (2) of Definition 2.9, we would conclude that this curve is equivalent to the concatenation of the line that joins $(-1,0)$ to $(0,0)$ with the line that joins $(0,0)$ to $(-1,0)$. This curve is cleary homotopic to a point and hence the curve that joins $(-1,0)$ to $(1,0)$ would be equivalent to a point.

DEFINITION 2.15 (Closed geodesics in a Riemannian orbifold). Let $\Sigma / W$ be a Riemannian orbifold. A closed geodesic in $\Sigma / W$ is defined as

(1) a sequence $0=t_{0}<\cdots<t_{n}=1$,

(2) non-trivial segments of geodesics $\gamma_{i}:\left[t_{i-1}, t_{i}\right] \rightarrow \Sigma, 1 \leq i \leq n$,

(3) elements $w_{i} \in W$ defined in a neighbourhood of $\gamma_{i}\left(t_{i}\right)$ for $1 \leq i \leq n$ such that $\gamma_{1}(0)=w_{n} \gamma_{n}(1), \gamma_{1}^{\prime}(0)=d w_{n} \gamma_{n}^{\prime}(1), w_{i} \gamma_{i}\left(t_{i}\right)=\gamma_{i+1}\left(t_{i}\right), d w_{i} \gamma_{i}^{\prime}\left(t_{i}\right)=\gamma_{i+1}^{\prime}\left(t_{i}\right)$, where $1 \leq i \leq n-1$.

Usually a closed geodesic in $\Sigma / W$ is denoted by $\left(\gamma_{i}, w_{i}\right)$.

In order to prove the existence of closed geodesics in each compact Riemannian good orbifold, it suffices to prove the existence of closed geodesics in each compact Riemannian good orbifold $\Sigma / W$, where $\Sigma$ is a complete connected Riemannian manifold and $W$ has infinite cardinality. Therefore, the next proposition gives an alternative proof of item (2) of Guruprasad and Haefliger's theorem [11, Theorem 5.1.1, Remark 5.1.2].

Proposition 2.16. Let $\Sigma$ be a connected complete Riemannian manifold and $W$ be an infinite discrete subgroup of isometries of $\Sigma$ whose action on $\Sigma$ is proper and such that $\Sigma / W$ is a compact good orbifold. Assume that there exists an element $w^{0} \in W$ that does not fix points (e.g. $w^{0}$ has infinite order). Then there exists a non-trivial closed geodesic in the Riemannian good orbifold $\Sigma / W$.

Proof. Let us first prove that $\inf _{y \in \Sigma} \mathrm{d}\left(y, w^{0} y\right)>0$. Assume on the contrary that there exists a sequence $\left\{x_{n}\right\}_{n \in \mathbb{N}}$ in $\Sigma$ such that $\lim _{n \rightarrow \infty} \mathrm{d}\left(x_{n}, w^{0} x_{n}\right)$ goes to zero. As $\Sigma / W$ is compact, there exists a sequence $\left\{g_{k}\right\}_{k \in \mathbb{N}}$ in $W$ and $y \in \Sigma$ such that

$$
\lim _{k \rightarrow \infty} g_{k} x_{k}=y \quad \text { and } \quad \lim _{k \rightarrow \infty} g_{k} w^{0} x_{k}=y
$$

up to subsequences. As the second limit coincides with $\lim _{k \rightarrow \infty} g_{k} w^{0} g_{k}^{-1} g_{k} x_{k}=y$ and the action of $W$ in $\Sigma$ is proper, we obtain that $g_{k} w^{0} g_{k}^{-1} \rightarrow g \in W_{y}$, and being the action $W$ discrete, it follows that there exists $k_{0} \in \mathbb{N}$ such that $g=g_{k_{0}} w^{0} g_{k_{0}}^{-1}$. Thus, $g_{k_{0}} w^{0} g_{k_{0}}^{-1} y=y$, but in this case $w^{0}$ fixes $g_{k_{0}}^{-1} y$ contradicting our hypothesis. Once we have $\inf _{y \in \Sigma} \mathrm{d}\left(y, w^{0} y\right)>0$, a similar argument proves that the infimum is attained at some point $x \in \Sigma$. Let $\gamma:[0,1] \rightarrow \Sigma$ be the geodesic minimising the distance from $x$ to $w^{0} x$, which exists because $\Sigma$ is complete. If we prove that $\tilde{\gamma}:[0,2] \rightarrow \Sigma$ given by

$$
\tilde{\gamma}(t)= \begin{cases}\gamma(t) & \text { if } t \in[0,1] \\ w^{0} \gamma(t-1) & \text { if } t \in(1,2]\end{cases}
$$


is a smooth geodesic, then its projection in $\Sigma / W$ will be a closed geodesic. Let $x^{\prime}=$ $\gamma\left(t^{\prime}\right)$ with $t^{\prime} \in(0,1)$. Then $\mathrm{d}\left(x^{\prime}, w^{0} x^{\prime}\right) \leq \mathrm{d}\left(x^{\prime}, w^{0} x\right)+\mathrm{d}\left(w^{0} x, w^{0} x^{\prime}\right)=\mathrm{d}\left(x^{\prime}, w^{0} x\right)+$ $\mathrm{d}\left(x, x^{\prime}\right)=\mathrm{d}\left(x, w^{0} x\right)$. As $\mathrm{d}\left(x, w^{0} x\right)$ attains the minimum of the translation length of $w^{0}$, the last inequality must be in fact an equality and $\tilde{\gamma}$ must be smooth in $t=1$. As it is smooth in the rest of points, we finally conclude that its projection is a closed geodesic of the orbifold $\Sigma / W$.

REMARK 2.17. It is interesting to note that if there existed an infinite torsion in finitely presented group, then it would be possible to construct an example of a compact Riemannian good orbifold $\Sigma / W$ (that is not a manifold) so that $W$ would not necessarily satisfy the condition of Proposition 2.16. Nevertheless, as far as the authors know, the existence of such type of group remains an open problem.

We now recall the definition of singular Riemannian foliation.

DEFINITION 2.18. A partition $\mathcal{F}$ of a complete Riemannian manifold $M$ by connected immersed submanifolds (the leaves) without self-intersections is said to be

(1) a singular foliation, if the module $\mathcal{X}_{F}$ of smooth vector fields on $M$ that are tangent at each point to the corresponding leaf acts transitively on each leaf. In other words, for each leaf $L$ and each $v \in T L$ with footpoint $p$, there is $X \in \mathcal{X}_{F}$ with $X(p)=v$;

(2) a singular Riemannian foliation, if it satisfies (1) and it is transnormal, i.e. every geodesic that is perpendicular at one point to a leaf remains perpendicular to every leaf that meets.

REMARK 2.19. Let $\mathcal{F}$ be an s.r.f. A leaf $L$ of $\mathcal{F}$ (and each point in $L$ ) is called regular if the dimension of $L$ is maximal, otherwise $L$ is called singular. If all the leaves of $\mathcal{F}$ have the same co-dimension $k$, then $\mathcal{F}$ turns out to be a Riemannian foliation of codimension $k$.

Typical examples of (singular) Riemannian foliations are the partition by orbits of an isometric action, by leaf closures of a Riemannian foliation (see [2] and [18]), examples constructed by suspension of homomorphisms (see [1] and [2]) and examples constructed by changes of metric and surgery (see [3]).

In what follows we prove that if $M$ is simply connected and $M / \mathcal{F}$ is a compact orfibold, then $M / \mathcal{F}$ admits a closed geodesic, even if $\mathcal{F}$ is an s.r.f. We recall that the Coxeter orbifold is an orbifold satisfying that for each $x \in \Sigma$ there exists an open neighbourhood $U$ of $x$ in $\Sigma$ such that the retriction of $W$ to $U$ is generated by reflections in a finite (possibly empty) set of totally geodesic hypersurfaces with the common point $x$ so that the topology induced in this group (from the group of isometries) is discrete and the action is proper.

THEOREM 2.20. Let $\mathcal{F}$ be a singular Riemannian foliation with closed embedded leaves on a complete Riemannian manifold $M$ with finite fundamental group. Assume that $M / \mathcal{F}$ is a compact orbifold. Then $M / \mathcal{F}$ admits a non-trivial closed geodesic.

Proof. If $M / \mathcal{F}$ is a compact orbifold that is a manifold, then the result follows from the theorem given by Lyusternik and Fet (see, for example, [13]). Therefore, from now on, we assume that $M / \mathcal{F}$ is a compact orbifold that is not a manifold.

First we will prove the case in which $M$ is simply connected. If $\mathcal{F}$ is a (regular) Riemannian foliation, according to Salem [18, Appendix D], there exists a surjective 
homomorphism between $\pi_{1}(M)$ and the fundamental group of the holonomy of the foliation, which coincides with the fundamental group of the orbifold $M / \mathcal{F}$. Therefore, the fundamental group of the orbifold $M / \mathcal{F}$ is trivial and hence cannot be a good orbifold (see [7, page 608]). Then the result follows from item (1) of Guruprasad and Haefliger's theorem [11]. If $\mathcal{F}$ is an s.r.f. and $M / \mathcal{F}$ is not developable, then the result also follows from item (1) of Guruprasad and Haefliger's theorem [11].

Now assume that $\mathcal{F}$ is an s.r.f. and $M / \mathcal{F}$ is a good orbifold. Then according to Lytchak [14, Theorem 1.8] $M / \mathcal{F}$ is a good Coxeter orbifold with regular leaves with trivial holonomy. In addition, $\mathcal{F}$ is an infinitesimal polar foliation, i.e. the restriction of the foliation to each slice is diffeomorphic, by the exponencial map, to an s.r.f. with sections (see definition below) on the tangent space of the slice. In other words, $\mathcal{F}$ is infinitesimally polar if the restriction of the foliation to each slice is diffeomorphic (by the composition of the exponencial map with a linear map) to an isoparametric foliation on an Euclidean space (see definition in [15]). Therefore we can adapt the proof given by Alexandrino and Töben [3, Theorem 1.6] to conclude that the topological space $|M / \mathcal{F}|$ is simply connected and for each chamber $C$, i.e. a connected component of $\Sigma-\left(\cup_{w \in W} \mathrm{Fix}_{w}\right)$, we have $M / \mathcal{F}=\Sigma / W=\bar{C}$, where $W$ is a cocompact discrete reflection group. Here $\mathrm{Fix}_{w}$ denotes the fixed-point set of $w$.

Note that each reflection $r \in W$ can be lifted to a dissecting reflection $\tilde{r}$ on the universal covering space $\tilde{\Sigma}$ of $\Sigma$, i.e. a reflection such that $\tilde{\Sigma}-$ Fix $_{\tilde{r}}$ has exactly two connected components. This is because every reflection in a simply connected space is dissecting. Let us denote $\tilde{W}$ the group generated by these dissecting reflections.

Claim The action of $\tilde{W}$ on $\tilde{\Sigma}$ is a cocompact action and $\Sigma / W=\tilde{\Sigma} / \tilde{W}$.

In order to prove the claim, let $\tilde{C}$ be a lift of $C$. Since $C$ is simply connected, $\bar{C}$ is simply connected and compact. We want to prove that for each point $\tilde{x} \in \tilde{\Sigma}$ the orbit $\tilde{W}(\tilde{x})$ meets $\bar{C}$ exactly once. First we want to prove that $\tilde{W}(\tilde{x})$ meets $\bar{C}$. Join $\tilde{x}$ to $\tilde{C}$ by a curve $\tilde{\alpha}$ and set $\alpha=\pi \tilde{\alpha}$, where $\pi: \tilde{\Sigma} \rightarrow \Sigma$ is the covering map. We may assume that $\alpha$ meets the singular stratification only in the walls and always transversally to them at points $\alpha\left(t_{i}\right)$. Since reflections send chambers to chambers, by reflections in the walls that contain the points $\alpha\left(t_{i}\right)$ we obtain $W(x) \cap \bar{C} \neq \emptyset$ for every $x \in \Sigma$. Therefore, by the reflections in the walls that contain the points $\tilde{\alpha}\left(t_{i}\right)$, we also conclude that $\tilde{W}(\tilde{x}) \cap \overline{\tilde{C}} \neq \varnothing$. Now we want to prove that $\tilde{W}(\tilde{x})$ meets $\tilde{C}$ only once. Note that for each reflection $r$ (and its lifts $\tilde{r}$ ) we have $\pi \tilde{r}=r \pi$. Therefore, for each $\tilde{w} \in \tilde{W}$ there exists $w$ such that $\pi \tilde{w}=w \pi$. The facts that each orbit of $W$ meets $\bar{C}$ exactly once and $\tilde{C}$ is homeomorphic to $C$ imply that $\tilde{W}(\tilde{x})$ meets $\overline{\tilde{C}}$ only once and this concludes the proof of the claim.

Now, since $\tilde{W}$ is a cocompact discrete dissecting reflection group, it follows from Hosaka [12] that $\tilde{W}$ is the finitely generated Coxeter group (for an algebraic definition of Coxeter group, see, for example, [12]). It is known that any finitely generated Coxeter group $\tilde{W}$ has a torsion-free subgroup of finite index (see [10, Proposition 1.4]). Therefore $\tilde{W}$ is finite or $\tilde{W}$ has an element of infinite order. In both cases we have seen in Proposition 2.16 that $M / \mathcal{F}=\Sigma / W=\tilde{\Sigma} / \tilde{W}$ admits a closed geodesic.

Finally, consider the case in which $M$ has finite fundamental group. Let $\tilde{M}$ be the universal covering of $M$. Then the foliation $(M, \mathcal{F})$ induces naturally a foliation $(\tilde{M}, \tilde{\mathcal{F}})$. As we have assumed that the fundamental group of $M$ is finite, it follows that $\tilde{M} / \tilde{\mathcal{F}}$ is compact and the leaves of $\tilde{\mathcal{F}}$ are closed and embedded. Thus, we can apply 
the first part of the proof to obtain a closed geodesic in $\tilde{M} / \tilde{\mathcal{F}}$ that projects to a closed geodesic in $M / \mathcal{F}$, as desired.

REMARK 2.21. One should note that, although the proofs of [3] are correct, there exists a misprint throughout this paper. Every time when one refers to the fundamental group of a pseudogroup in the proofs, one is, in fact, talking about the fundamental group of the topological space $|M / \mathcal{F}|$. Moreover, in [12], the author calls reflections what we call dissecting reflections. This is the reason why we have to lift the reflections in $\tilde{\Sigma}$ in order to apply Hosaka's theorem [12].

When $M$ is simply connected and the leaves of $\mathcal{F}$ are closed and embedded, there are (at least) two special classes of s.r.fs. such that $M / \mathcal{F}$ is an orbifold.

The first one is the class of singular Riemannian foliations without horizontal conjugate points. This concept was introduced by Lytchak and Thorbergsson [15] and generalises the definition of variationally complete actions. $\mathcal{F}$ is without horizontal conjugate points if the following is true: for all leaves $L$ and all geodesics $\gamma$ meeting $L$ perpendicularly, any $L$-Jacobi field $J$ along $\gamma$ that is tangent to a leaf of $\mathcal{F}$ different from $L$ is tangent to all leaves passing through $\gamma$. It follows from Lytchak [14, Theorems 1.2 and 1.8] that $M / \mathcal{F}$ is a Riemannian Coxeter orbifold, if $M$ is simply connected and the leaves of $\mathcal{F}$ are closed and embedded (see also [16, Theorem 1.7]).

The other class is made up of s.r.fs. with sections. This concept was introduced by Alexandrino [1]. Typical examples of s.r.f. with sections are the partition by orbits of a polar action, isoparametric foliations on space forms (some of them with inhomogeneous leaves) and partitions by parallel submanifolds of an equifocal submanifold (see [19] and [20]).

An s.r.f. admits sections if for each regular point $p$, the set $\Sigma:=\exp \left(v_{p} L\right)$ (section) is a complete immersed submanifold that meets each leaf orthogonally.

It was proved by Alexandrino and Töben [3, Theorem 1.6] that $M / \mathcal{F}$ is a Coxeter orbifold if $M$ is simply connected and the leaves of $\mathcal{F}$ are closed and embedded (see also [4]).

Recently, Lytchak [14, Theorem 1.2] proved that if $\mathcal{F}$ is as s.r.f. on a simply connected complete Riemannian manifold such that $\mathcal{F}$ admits sections or $\mathcal{F}$ has no horizontal conjugate points, then the leaves of $\mathcal{F}$ are closed and embedded.

The above discussion and Theorem 2.20 imply the next corollary.

COROLlary 2.22. Let $\mathcal{F}$ be a singular Riemannian foliation on a complete Riemannian manifold $M$ with finite fundamental group such that $M / \mathcal{F}$ is compact. Assume that $\mathcal{F}$ admits sections or $\mathcal{F}$ has no horizontal conjugate points. Then $M / \mathcal{F}$ admits a non-trivial closed geodesic.

REMARK 2.23. The above corollary and Myers' theorem imply that if $\mathcal{F}$ is an s.r.f. that admits sections or $\mathcal{F}$ has no horizontal conjugate points on a complete Riemannian manifold with Ric $\geq k>0$ (e.g. symmetric spaces of compact type), then $M / \mathcal{F}$ admits a closed geodesic. Therefore we have the existence of closed geodesics in the orbit spaces of polar and variationally complete actions in symmetric spaces of compact type, the usual space where these actions are studied.

3. Riemannian foliations and shortening process. In this section we study the shortening process with respect to Riemannian foliations and infer some results. 
3.1. Equifocality of Riemannian foliations. In this subsection we fix some definitions and recall an important property of Riemannian foliations, namely the equifocality.

A Bott or basic connection $\nabla$ of a foliation $\mathcal{F}$ is a connection of the normal bundle of the leaves with $\nabla_{X} Y=[X, Y]^{\nu \mathcal{F}}$ whenever $X \in \mathcal{X}_{F}$ and $Y$ is a vector field of the normal bundle $\nu \mathcal{F}$ of the foliation. Here the superscript $\nu \mathcal{F}$ denotes projection onto $\nu \mathcal{F}$ and $\mathcal{X}_{F}$ denotes the module of smooth vector fields on $M$ that are tangent at each point to the corresponding leaf.

A normal foliated vector field is a normal field parallel with respect to the Bott connection. If we consider a local submersion $f$ that describes the plaques of $\mathcal{F}$ in the neighbourhood of a point of $L$, then a normal foliated vector field is a normal projectable/basic vector field with respect to $f$.

A fundamental property of Riemannian foliations, called equifocality, says that if $\xi$ is a normal parallel vector field (with respect to the Bott connection) along a curve $\beta:[0,1] \rightarrow L$, then the curve $t \mapsto \exp _{\beta(t)}(\xi)$ is contained in the leaf $L_{\exp _{\beta(0)}(\xi)}$.

This property still holds even for s.r.fs. and implies that one can reconstruct the (singular) foliation by taking all parallel submanifolds of a (regular) leaf with trivial holonomy (see [5]).

The equifocality allows us to introduce the concept of parallel transport (with respect to the Bott connection) of horizontal segments of geodesic.

Definition 3.1. Let $\beta:[a, b] \rightarrow L$ be a piecewise smooth curve and $\gamma:[0,1] \rightarrow M$ a segment of horizontal geodesic such that $\gamma(0)=\beta(a)$. Let $\xi_{0}$ be a vector of the normal space $v_{\beta(a)} L$ such that $\exp _{\gamma(0)}\left(\xi_{0}\right)=\gamma(1)$ and $\xi:[a, b] \rightarrow \nu L$ the parallel transport of $\xi_{0}$ with respect to the Bott connection along $\beta$. We define $\|_{\beta}(\gamma):=\hat{\gamma}$, where $\hat{\gamma}$ : $[0,1] \rightarrow M$ is the segment of geodesic given by $s \rightarrow \hat{\gamma}(s)=\exp _{\beta(b)}(s \xi(b))$. We also set $\eta(\gamma, \beta):=\hat{\beta}$, where $\hat{\beta}$ is the curve contained in $L_{\gamma(1)}$ defined as $s \rightarrow \hat{\beta}(s)=\exp _{\beta(s)}(\xi(s))$.

Owing to the equifocality of $\mathcal{F}$, we can give an alternative definition of holonomy map of a Riemannian foliation. Let $\beta:[0,1] \rightarrow L$ be a piecewise smooth curve and $S_{\beta(i)}:=\left\{\exp _{\beta(i)}(\xi) \mid \xi \in v_{\beta(i)} L,\|\xi\|<\epsilon\right\}$ the slice at $\beta(i)$, for $i=0,1$. Then a holonomy map $\varphi_{[\beta]}: S_{\beta(0)} \rightarrow S_{\beta(1)}$ is defined as $\varphi_{[\beta]}(x):=\|_{\beta} \gamma(r)$, where $\gamma:[0, r] \rightarrow S_{\beta(0)}$ is the minimal segment of geodesic that joins $\beta(0)$ to $x$. Since the Bott connection is locally flat, the parallel transport depends only on the homotopy class $[\beta]$.

Using the holonomy map of a Riemannian foliation we can define horizontal periodic geodesics as follows.

Definition 3.2. Let $\mathcal{F}$ be a Riemannian foliation. A geodesic $\gamma$ is called $\mathcal{F}$ horizontal periodic if

(a) $\gamma$ is horizontal, i.e. is orthogonal to the leaves of $\mathcal{F}$,

(b) there exists $0<t_{1}$ such that $\gamma\left(t_{1}\right) \in L_{\gamma(0)}$,

(c) there exists a holonomy map $\varphi_{[\beta]}$ such that $d \varphi_{[\beta]} \gamma^{\prime}(0)=\gamma^{\prime}\left(t_{1}\right)$.

If $t_{1}$ is the smallest positive number that satisfies (b) and (c), then $t_{1}$ is called the period of $\gamma$.

REMARK 3.3. By the equifocality of Riemannian foliations we can deduce that for each fixed $s$ and each $n \in \mathbb{Z}$ we have

(a) $\gamma\left(n t_{1}+s\right) \in L_{\gamma(s)}$;

(b) there exists a holonomy map $\varphi_{\left[\beta_{n}\right]}$ such that $d \varphi_{\left[\beta_{n}\right]} \gamma^{\prime}(s)=\gamma^{\prime}\left(n t_{1}+s\right)$. 
Furthermore, if $M$ is compact and the leaves of $\mathcal{F}$ are compact, then for each closed geodesic of the Riemannian orbifold $M / \mathcal{F}$ there exists a horizontal periodic geodesic and vice versa.

We also need the notation below, which turns out to be very convenient as to describing the curve-shortening procedure.

Definition 3.4. Let $\mathcal{F}$ be a Riemannian foliation on $(M, \mathrm{~g})$ and $\alpha$ and $\beta$ be two piecewise smooth curves $\alpha:[a, b] \rightarrow M$ and $\beta:[a, b] \rightarrow M$ such that the endpoints of $\alpha$ belong to the same leaf $L_{\alpha(a)}$ and the image of $\beta$ is contained in $L_{\alpha(a)}$ with $\beta(a)=\alpha(a)$ and $\beta(b)=\alpha(b)$. Then we say that a pair $\left(\alpha, \varphi_{[\beta]}\right)$ is an $\mathcal{F}$-closed pair, where $\varphi_{[\beta]}$ is the holonomy map in $\mathcal{F}$ associated to $\beta$. In addition, a pair $\left(\alpha, \varphi_{[\beta]}\right)$ is called $\mathcal{F}$-well closed pair if $\alpha$ is regular in $\alpha(a)$ and $\alpha(b)$, and if $d \varphi_{[\beta]} d f_{a} \alpha^{\prime}(a)=d f_{b} \alpha^{\prime}(b)$, where $f_{i}: \operatorname{Tub}\left(P_{\alpha(i)}\right) \rightarrow S_{\alpha(i)}$ is a submersion that describes the plaques in the neighbourhood of $\alpha(i)$ for $i=a, b$.

Note that the horizontal periodic geodesic $\gamma$ is a well-closed pair $\left(\gamma, \varphi_{[\beta]}\right)$.

3.2. Shortening process. In this subsection we construct the shortening process with respect to a Riemannian foliation.

REMARK 3.5 (Conventions). We will use two different concatenations of curves. We will denote by $*$ the curve obtained as the union of two curves $\alpha_{1}:[a, b] \rightarrow M$ and $\alpha_{2}:[b, c] \rightarrow M$, that is, the curve $\alpha_{1} * \alpha_{2}:[a, c] \rightarrow M$ that coincides with $\alpha_{1}$ and $\alpha_{2}$ in $[a, b]$ and $[b, c]$, respectively. On the other hand, we will denote by $\star$ the concatenation of two curves $\alpha_{1}, \alpha_{2}:[a, b] \rightarrow M$, that is, the curve in $[a, b]$ such that $\alpha_{2} \star \alpha_{1}(s)=\alpha_{1}(2 s-$ $a)$ in $[a, a+(b-a) / 2]$ and $\alpha_{2} \star \alpha_{1}(s)=\alpha_{2}(2 s-b)$ in $[a+(b-a) / 2, b]$. Moreover, given the curve $\alpha:[a, b] \rightarrow M$, we will denote by $\alpha^{-1}:[a, b] \rightarrow M$ the curve defined as $\alpha^{-1}(s)=\alpha(b+a-s)$.

From now on, we assume that $\mathcal{F}$ is a Riemannian foliation with compact leaves on a compact Riemannian manifold $M$.

We will see in the following that it is possible to assign a horizontal piecewise $\mathcal{F}$-periodic geodesic to a given $\mathcal{F}$-closed pair $\left(\alpha, \varphi_{[\beta]}\right)$ (see Definition 3.4). This process involves several difficulties up to its definition. First we note that there exists a radius $\rho_{0}>0$ satisfying the following:

(i) It is smaller than the injectivity radius of every point.

(ii) The balls $B\left(x, \rho_{0}\right)$ are always contained in a trivial neighbourhood.

(iii) There exists a unique minimising horizontal geodesic between every point $x$ and every plaque for the trivial neighbourhood of (ii) at a distance lower than $\rho_{0}$.

3.2.1. $\hat{P}$-process. We are now ready to define the shortening process. Fix a real number $K>0$ and consider an $\mathcal{F}$-closed pair $\left(\alpha, \varphi_{[\beta]}\right)$ as in Definition 3.4 such that $E(\alpha) \leq K$. Given a partition

$$
a=l_{0}<l_{1}<\cdots<l_{k}=b
$$

such that $l_{i}-l_{i-1}<\frac{\rho_{0}^{2}}{K}$ for $i=1, \ldots, k$, Holder's inequality implies that

- $d\left(\alpha\left(l_{i-1}\right), \alpha\left(l_{i}\right)\right)<\rho_{0}$,

- $\left.\alpha\right|_{\left[l_{i-1}, l_{i}\right]}$ is contained in a trivial neighbourhood of $\mathcal{F}$, 
- there exists a unique minimising horizontal geodesic $\tilde{\gamma}_{i}:\left[l_{i-1}, l_{i}\right] \rightarrow M$ joining $\alpha\left(l_{i-1}\right)$ and the plaque in the trivial neighbourhood containing $\alpha\left(l_{i}\right)$ and that satisfies $E\left(\tilde{\gamma}_{i}\right) \leq$ $E\left(\left.\alpha\right|_{\left[l_{i-1}, l_{i}\right]}\right)$.

Therefore, we can construct a piecewise 'disconnected' horizontal geodesic from the curve $\alpha$. Now we will use the trivial holonomy in every trivial neighbourhood to obtain a connected piecewise horizontal geodesic as a holonomy between the endpoints.

For $1 \leq i \leq k$, let $\hat{\gamma}_{i, i}$ be the minimising segment of geodesic orthogonal to the plaque $P_{\alpha\left(l_{i}\right)}$ such that $\hat{\gamma}_{i, i}\left(l_{i-1}\right)=\alpha\left(l_{i-1}\right)$ and $\hat{\gamma}_{i, i}\left(l_{i}\right) \in P_{\alpha\left(l_{i}\right)}$. Let $\hat{\beta}_{i, i}$ be a curve from $\left[l_{i-1}, l_{i}\right]$ into $P_{\alpha\left(l_{i}\right)}$ such that $\hat{\beta}_{i, i}\left(l_{i-1}\right)=\hat{\gamma}_{i, i}\left(l_{i}\right)$ and $\hat{\beta}_{i, i}\left(l_{i}\right)=\alpha\left(l_{i}\right)$. Assume that $\hat{\beta}_{n-1, j}$ and $\hat{\gamma}_{n, j+1}$ are defined, then $\hat{\gamma}_{n, j}:=\|_{\hat{\beta}_{n-1, j}^{-1}}\left(\hat{\gamma}_{n, j+1}\right)$ and $\hat{\beta}_{n, j}:=\eta\left(\hat{\gamma}_{n, j}, \hat{\beta}_{n-1, j}\right)$. Apply this process inductively for $n=2, \ldots, k$ and $j=n-1, \ldots, 1$. Finally, define $\tilde{\beta}:=\hat{\beta}_{k, 1} * \cdots * \hat{\beta}_{k, k}$, the piecewise horizontal geodesic $\hat{\gamma}=\hat{\gamma}_{1,1} * \hat{\gamma}_{2,1} \cdots * \hat{\gamma}_{k, 1}$, and the holonomy of the endpoints by the curve $\hat{\beta}=\tilde{\beta}^{-1} \star \beta$. Summing up, given the $\mathcal{F}$-closed pair $\left(\alpha, \varphi_{[\beta]}\right)$ and a family of nodes $a=l_{0}<l_{1}<\cdots<l_{k}=b$ such that $l_{i}-l_{i-1}<\frac{\rho_{0}^{2}}{K}$ for $i=1, \ldots, k$, we have obtained an $\mathcal{F}$-closed pair $\hat{P}\left(\alpha, \varphi_{[\beta]}\right)=\left(\hat{\gamma}, \varphi_{[\hat{\beta}]}\right)$ such that $\hat{\gamma}$ is a piecewise horizontal geodesic with $E(\hat{\gamma}) \leq K$ and $\hat{\beta}$ is a curve in $L_{\hat{\gamma}(a)}$ that joins the endpoints of $\hat{\gamma}$.

3.2.2. The double shortening map. As usual we will alternate two families of nodes in the shortening process to obtain a smooth curve in the limit. Choose two partitions $\left\{t_{i}\right\}$ and $\left\{\tau_{i}\right\}$ with $i=1, \ldots, k$ such that

$$
\tau_{0}=\tau_{k}-1<t_{0}=0<\tau_{1}<t_{1}<\tau_{2}<t_{2}<\cdots<\tau_{k}<t_{k}=1,
$$

and $t_{i}-t_{i-1}, \tau_{i}-\tau_{i-1}<\frac{\rho_{0}^{2}}{K}$ for $i=1, \ldots, k$. Given an $\mathcal{F}$-closed pair $\left(\alpha, \varphi_{[\beta]}\right)$ as in the preceding subsection with $\alpha$ defined in [0,1], we can apply the $\hat{P}$-process with the partition $0=t_{0}<\cdots<t_{k}=1$, obtaining a horizontal piecewise geodesic $\hat{\gamma}$ and a curve $\hat{\beta}$ in the leaf $L_{\hat{\gamma}(0)}$ joining the endpoints of $\hat{\gamma}$. Now we can extend $\hat{\gamma}$ by parallel transport to $\left[\tau_{0}, 0\right]$ as follows:

$$
\hat{\gamma}(t):=\|_{\hat{\beta}^{-1}}\left(\left.\hat{\gamma}\right|_{\left[\tau_{k}, 1\right]}\right)(t+1) .
$$

Moreover, we can bring the holonomy $\varphi_{[\hat{\beta}]}$ along $\left.\hat{\gamma}\right|_{\left[\tau_{0}, 0\right]}$ using the endpoint map $\eta$ (see Definition 3.1) obtaining a holonomy $\varphi_{[\bar{\beta}]}$ in the leaf of $\hat{\gamma}\left(\tau_{0}\right)$ with $\bar{\beta}(0)=\hat{\gamma}\left(\tau_{0}\right)$ and $\bar{\beta}(1)=\hat{\gamma}\left(\tau_{k}\right)$. We can again apply the $\hat{P}$-process to the curve $\hat{\gamma}:\left[\tau_{0}, \tau_{k}\right] \rightarrow M$ and the holonomy $\varphi_{[\bar{\beta}]}$ obtaining $\hat{P}\left(\hat{\gamma}, \varphi_{[\bar{\beta}]}\right)=\left(\gamma_{0}, \varphi_{\left[\bar{\beta}_{0}\right]}\right)$. Finally, we extend the curve $\gamma_{0}$ to $\left[\tau_{k}, 1\right]$ as

$$
\gamma_{0}(t):=||_{\bar{\beta}_{0}}\left(\left.\gamma_{0}\right|_{\left[\tau_{0}, 0\right]}\right)(t-1)
$$

and consider in the leaf of $\gamma_{0}(0)$ the holonomy given by the endpoint map $\eta$ of $\bar{\beta}_{0}$ along $\left.\gamma_{0}\right|_{\left[\tau_{0}, 0\right]}$ obtaining an $\mathcal{F}$-closed pair $\left(\gamma_{0}, \varphi_{\left[\beta_{0}\right]}\right)$. Therefore, we have obtained a double shortening map, that is, $P_{0}\left(\alpha, \varphi_{[\beta]}\right)=\left(\gamma_{0}, \varphi_{\left[\beta_{0}\right]}\right)$.

\subsection{Main propositions.}

Proposition 3.6. Let $\left(\alpha, \varphi_{[\beta]}\right)$ be an $\mathcal{F}$-closed pair (with $\alpha:[0,1] \rightarrow M$ ) such that $E(\alpha) \leq K$ and $P_{0}\left(\alpha, \varphi_{[\beta]}\right)=\left(\gamma_{0}, \varphi_{\left[\beta_{0}\right]}\right)$. Then $E\left(\left.\gamma_{0}\right|_{[0,1]}\right) \leq E(\alpha)$ with equality if and only if $\alpha$ is a horizontal periodic geodesic. 
Proof. We have already observed in Subsection 3.2.1 that a shortening $\hat{P}\left(\alpha, \varphi_{[\beta]}\right)=$ $\left(\hat{\gamma}, \varphi_{[\hat{\beta}]}\right)$ satisfies $E(\hat{\gamma}) \leq E(\alpha)$. As the geodesic segments of $\hat{\gamma}$ are the unique minimising geodesics joining the initial point with the plaque of the endpoint, the equality holds if and only if $\alpha$ is a piecewise geodesic with nodes $t_{0}, \ldots, t_{n-1}$, and in this case $\hat{\gamma}=\alpha$. In the $P_{0}$-process we apply twice the $\hat{P}$-process. As we change the nodes and $E\left(\left.\hat{\gamma}\right|_{\left[\tau_{0}, \tau_{k}\right]}\right)=$ $E\left(\left.\hat{\gamma}\right|_{[0,1]}\right)$, the energy of $\gamma_{0}$ remains the same if and only if $\alpha$ is a geodesic such that the extension to $\left[\tau_{0}, 0\right]$ by the parallel transport along $\beta$ gives a geodesic $\gamma_{0}$ in $\left[\tau_{0}, 1\right]$.

In the following, we will say that a curve $\alpha:[a, b] \rightarrow M$ is $\mathcal{F}$-closed if the endpoints are in the same leaf of $\mathcal{F}$. We say that two $\mathcal{F}$-closed curves are $\mathcal{F}$-homotopic if there exists a homotopy between them by $\mathcal{F}$-closed curves.

The fact that the restriction of the considered curves to the partitions $\left\{t_{i}\right\}$ and $\left\{\tau_{i}\right\}$ are contained in trivial neighbourhoods of $\mathcal{F}$ and the equifocality of $\mathcal{F}$ imply the next lemma.

Lemma 3.7. Let $\left(\alpha, \varphi_{[\beta]}\right)$ be an $\mathcal{F}$-closed pair such that $E(\alpha) \leq K$ and $\hat{P}\left(\alpha, \varphi_{[\beta]}\right)=$ $\left(\hat{\gamma}, \varphi_{[\hat{\beta}]}\right)$. Then $\hat{\gamma}$ is $\mathcal{F}$-homotopic to $\alpha$.

Proposition 3.8. Let $\left(\alpha, \varphi_{[\beta]}\right)$ be an $\mathcal{F}$-closed pair (with $\alpha:[0,1] \rightarrow M$ ) such that $E(\alpha) \leq K$ and $P_{0}\left(\alpha, \varphi_{[\beta]}\right)=\left(\gamma_{0}, \varphi_{\left[\beta_{0}\right]}\right)$. Then $\gamma_{0}$ is $\mathcal{F}$-homotopic to $\alpha$.

Proof. By applying Lemma 3.7 we obtain that $\alpha$ is $\mathcal{F}$-homotopic to the first shortening $\hat{\gamma}$. As we extend $\hat{\gamma}$ with the holonomy $\varphi_{[\hat{\beta}]}$, we have $\hat{\gamma}(t)$ and $\hat{\gamma}(t+1)$ in the same leaf for $t \in\left[\tau_{0}, 0\right]$. It also follows from Lemma 3.7 that there exists a map $H$ (that we call $\mathcal{F}$ homotopy) defined in $\left[\tau_{0}, \tau_{k}\right] \times[0,1]$ such that

(1) $H\left(\tau_{k}, s\right) \in L_{H\left(\tau_{0}, s\right)}$ for each $s \in[0,1]$,

(2) $H(\cdot, 0)=\left.\hat{\gamma}\right|_{\left[\tau_{0}, \tau_{k}\right]}$ and $H(\cdot, 1)=\left.\gamma_{0}\right|_{\left[\tau_{0}, \tau_{k}\right]}$.

By transporting horizontal segments of geodesics, the $\mathcal{F}$-homotopy $H$ can be chosen to admit an extension to $\left[\tau_{0}, 1\right] \times[0,1]$ so that $H(1, s) \in L_{H(0, s)}$ for each $s$. Therefore $\left.\gamma_{0}\right|_{[0,1]}$ is $\mathcal{F}$-homotopic to $\left.\hat{\gamma}\right|_{[0,1]}$ and, hence, $\mathcal{F}$-homotopic to $\alpha$.

We will denote by $\Pi_{1}$ and $\Pi_{2}$, respectively, the first and the second projections of an $\mathcal{F}$-closed pair. Given a closed curve, if nothing is said, we will assume that it is an $\mathcal{F}$-closed pair considering the trivial holonomy.

Proposition 3.9. Let $\alpha:[0,1] \rightarrow M$ be a closed curve with $E(\alpha) \leq K$. Then a subsequence of $\Pi_{1} \circ P_{0}^{n} \alpha$ converges uniformly to a (possibly trivial) horizontal periodic geodesic.

Proof. Each curve $\Pi_{1} \circ P_{0}^{n} \alpha$ is a horizontal periodic piecewise geodesic with nodes $\Pi_{1} \circ P_{0}^{n} \alpha\left(\tau_{1}\right), \ldots, \Pi_{1} \circ P_{0}^{n} \alpha\left(\tau_{k}\right)$. Note that each such curve may be identified with a $k$-tuple $\left(\Pi_{1} \circ P_{0}^{n} \alpha\left(\tau_{1}\right), \ldots, \Pi_{1} \circ P_{0}^{n} \alpha\left(\tau_{k}\right)\right) \in M^{k}:=M \times \cdots \times M$. Since $M^{k}$ is compact, a subsequence of these nodes converges to some $\left(p_{1}, \ldots, p_{k}\right) \in M^{k}$ and by the continuity of the exponential map, a subsequence $\left\{\gamma_{m}\right\}_{m \in \mathbb{N}}$ of $\Pi_{1} \circ P_{0}^{n} \alpha$ converges uniformly towards the horizontal piecewise geodesic $\gamma_{0}$ with nodes $\gamma_{0}\left(\tau_{i}\right)=p_{i}$ such that

$$
\left(\gamma_{m+1}, \varphi_{\left[\beta_{m+1}\right]}\right)=P_{0}^{\mu(m)}\left(\gamma_{m}, \varphi_{\left[\beta_{m}\right]}\right)
$$

with $\mu(m) \geq 1$.

We will see that the holonomies $\varphi_{\left[\beta_{m}\right]}$ admit a 'constant' subsequence in a certain sense. 
According to Molino [18, Lemma 3.7] we can choose a radius $\epsilon<1$ so that

- the tubular neighbourhood $\operatorname{Tub}_{\epsilon}\left(L_{\gamma_{0}(0)}\right)$ is saturated by leaves;

- for all $x \in L_{\gamma_{0}(0)}$ the slice $S_{x}$ (of radius $\epsilon$ ), defined as

$$
S_{x}:=\left\{\exp _{x}(\xi) \mid \xi \in \nu P_{x},\|\xi\|<\epsilon\right\},
$$

is transversal to the foliation;

- if $\tilde{L}$ is a leaf in $\operatorname{Tub}_{\epsilon}\left(L_{\gamma_{0}(0)}\right)$, then the all points of $\tilde{L}$ are at the same distance from $L$;

- for each $x \in L_{\gamma_{0}(0)}$ there exists a plaque $P_{x}$ such that $\pi^{-1}\left(P_{x}\right)$ is a simple open set, where $\pi: \operatorname{Tub}_{\epsilon}\left(L_{\gamma_{0}(0)}\right) \rightarrow L_{\gamma_{0}(0)}$ is the radial projection.

Choose $N_{0}$ such that if $m>N_{0}$, then $L_{\gamma_{m}(0)} \subset \operatorname{Tub}_{\frac{\epsilon}{2}}\left(L_{\gamma_{0}(0)}\right)$. Let $\beta_{m}$ be a representative for the holonomy class $\Pi_{2} \circ P_{0}^{m} \alpha \in L_{\gamma_{m}}(0)$ and define $\tilde{\beta}_{m}:=\pi\left(\beta_{m}\right) \in$ $L_{\gamma_{0}(0)}$, where $\pi: \operatorname{Tub}_{\frac{\epsilon}{2}}\left(L_{\gamma_{0}(0)}\right) \rightarrow L_{\gamma_{0}(0)}$ is the radial projection.

Our choice of $\epsilon$, the fact that the holonomy group of each leaf is finite and properties of the holonomy maps imply the next lemma.

LEMMA 3.10. In the above situation

(a) there exists a holonomy $\varphi_{\left[\beta_{0}\right]}$ in $L_{\gamma_{0}(0)}$ such that $\left(\gamma_{0}, \varphi_{\left[\beta_{0}\right]}\right)$ is a well closed pair,

(b) there exists a subsequence $\tilde{\beta}_{m_{i}}$ such that $\varphi_{\left[\beta_{0}\right]}=\varphi_{\left[\tilde{\beta}_{m i}\right]}: S_{\gamma_{0}(0)} \rightarrow S_{\gamma_{0}(1)}$.

For the sake of simplicity we will denote the subsequence $m_{i}$ by $m$. It is easy to see that

$$
E\left(\gamma_{m}\right)=\sum_{i=1}^{k} \frac{\operatorname{dist}\left(\gamma_{m}\left(\tau_{i-1}\right), \gamma_{m}\left(\tau_{i}\right)\right)^{2}}{2\left(\tau_{i}-\tau_{i-1}\right)},
$$

and then $\lim _{m \rightarrow \infty} E\left(\gamma_{m}\right)=E\left(\gamma_{0}\right)$. Therefore,

$$
\begin{aligned}
E\left(\gamma_{0}\right) & =\lim _{m \rightarrow \infty} E\left(\gamma_{m+1}\right)=\lim _{m \rightarrow \infty} E\left(\Pi_{1} \circ P_{0}^{\mu(m)}\left(\gamma_{m}, \varphi_{\left[\beta_{m}\right]}\right)\right) \\
& \leq \lim _{m \rightarrow \infty} E\left(\Pi_{1} \circ P_{0}\left(\gamma_{m}, \varphi_{\left[\beta_{m}\right]}\right)\right) \leq \lim _{m \rightarrow \infty} E\left(\gamma_{m}\right)=E\left(\gamma_{0}\right),
\end{aligned}
$$

where we have used Proposition 3.6. We conclude from the above equality that

$$
\lim _{m \rightarrow \infty} E\left(\Pi_{1} \circ P_{0}\left(\gamma_{m}, \varphi_{\left[\beta_{m}\right]}\right)\right)=E\left(\gamma_{0}\right) .
$$

The fact that minimal segments of geodesics depend smoothly on their endpoints, $\varphi_{\left[\beta_{0}\right]}=\varphi_{\left[\tilde{\beta}_{m}\right]}$, and that the energy is not changed by parallel transport of horizontal segments imply the next lemma.

LeMma 3.11. $E\left(\Pi_{1} \circ P_{0}\left(\gamma_{m}, \varphi_{\left[\beta_{m}\right]}\right)\right)$ converges to $E\left(\Pi_{1} \circ P_{0}\left(\gamma_{0}, \varphi_{\left[\beta_{0}\right]}\right)\right)$.

Lemma 3.11 and (3.4) imply

$$
E\left(\Pi_{1} \circ P_{0}\left(\gamma_{0}, \varphi_{\left[\beta_{0}\right]}\right)\right)=\lim _{m \rightarrow \infty} E\left(\Pi_{1} \circ P_{0}\left(\gamma_{m}, \varphi_{\left[\beta_{m}\right]}\right)\right)=E\left(\gamma_{0}\right),
$$

and from Proposition 3.6 we conclude that $\gamma_{0}$ is a horizontal periodic geodesic.

Proposition 3.12. Let $\mathcal{F}$ be a Riemannian foliation with compact leaves on a compact Riemannian manifold $M$. Assume that there exists a loop $\alpha$ in $M$ that is not free homotopic to any loop contained in any leaf. Then a subsequence of iterations of double shortening of $\alpha$ converges to a non-trivial horizontal periodic geodesic. 
REMARK 3.13. We observe that the condition of the last result is satisfied, for example, when there is an element of the fundamental group of $M$ with infinite order and the fundamental group of each leaf is finite.

Proof. Let $\alpha$ be a loop of $M$ that is not free homotopic to any loop contained in any leaf of $\mathcal{F}$. According to Proposition 3.9 there exists a subsequence $\left\{\gamma_{n}\right\}_{m \in \mathbb{N}}$ of $\Pi_{1} P_{0}^{m}(\alpha)$ that converges to a (possibly trivial) horizontal geodesic $\gamma^{0}$. Assume by contradiction that $\gamma^{0}=y$, i.e. $\gamma^{0}$ is trivial.

Consider $n$ big enough such that $\beta_{n}$ and $\gamma_{n}$ are in the same tubular neighbourhood $\operatorname{Tub}\left(L_{y}\right)$. By the radial projection in the axis $L_{y}$ we can construct a curve $\hat{\delta} \subset L_{y}$ such that $\hat{\delta}$ is free homotopic to $\beta_{n} \star \gamma_{n}$. Since $\beta_{n} \star \gamma_{n}$ is free homotopic to $\alpha$ (see Proposition 3.8 ), we conclude that $\alpha$ is free homotopic to $\hat{\delta}$. This contradicts the hypothesis. Therefore $\gamma^{0}$ is a non-trivial horizontal periodic geodesic.

The above proposition and Remark 2.7 imply the next result.

Corollary 3.14. Let $\Sigma / W$ be a Riemannian compact orbifold. Assume that the fundamental group of the topological space $|\Sigma / W|$ is non-trivial. Then there exists a non-trivial closed geodesic in the orbifold $\Sigma / W$.

REMARK 3.15. When $\Sigma / W$ is a good orbifold, one can simplify the construction of the double shortening map and get a map $P_{0}(\alpha, w)=(\gamma, w)$, where $(\alpha, w)$ and $(\gamma, w)$ are closed curves of $\Sigma / W$. In particular, if $\alpha$ is a curve that joints $x$ to $w^{0} x$ (where $w^{0}$ is an isometry that does not fix points), then there exist a subsequence $\left\{\gamma_{m}\right\}_{m \in \mathbb{N}}$ of $\left(\gamma_{n}, w\right)=P_{0}^{n}(\alpha, w)$ and a sequence $\left\{k_{m}\right\}_{m \in \mathbb{N}}$ in $W$ such that $\left\{k_{m} \gamma_{m}\right\}_{m \in \mathbb{N}}$ converges to a non-trivial closed geodesic $\left(\gamma_{0}, \tilde{w}\right)$. This gives us another proof of Proposition 2.16.

ACKNOWLEDGEMENTS. The authors are grateful to Dr. Alexander Lytchak for very useful suggestions.

\section{REFERENCES}

1. M. M. Alexandrino, Singular Riemannian foliations with sections, Illinois J. Math. 48(4) (2004), 1163-1182.

2. M. M. Alexandrino, Proofs of conjectures about singular Riemannian foliations, Geom. Dedicata 119(1) (2006), 219-234.

3. M. M. Alexandrino and D. Töben, Singular Riemannian foliations on simply connected spaces, Differ. Geom. Appl. 24(4) (2006), 383-397.

4. M. M. Alexandrino, Singular holonomy of singular Riemannian foliations with sections, Mat. Contemp. 33 (2007), 23-54.

5. M. M. Alexandrino and D. Töben, Equifocality of singular Riemannian foliations, Proc. Amer. Math. Soc. 136(9) (2008), 3271-3280.

6. M. M. Alexandrino, Desingularization of singular Riemannian foliation, Geom. Dedicata 149(1) (2010), 397-416.

7. M. R. Bridson and A. Haefliger, Metric spaces of non-positive curvature, Grundlehren der Mathematischen Wissenschaften, vol. 319 (Springer, New Mexico, 1999).

8. N. Bourbaki, Éléments de mathématique. Groupes et algèbres de Lie. Chapitres 4, 5 et 6. Actualités Scientifiques et Industrielles, No. 1337 (Hermann, Paris, 1968). 2000).

9. J. J. Duistermaat and J. A. C. Kolk, Lie groups (Springer-Verlag, Berlin, Heidelberg,

10. C. Gonciulea, Infinite Coxeter groups virtually surject onto Z, Comment. Math. Helv. 72(2) (1997), 257-265.

11. K. Guruprasad and A. Haefliger, Closed geodesics on orbifolds, Topology 45(3) (2006), 611-641. 
12. T. Hosaka, Reflection groups of geodesic spaces and Coxeter groups, Topol. Appl. 153 (2006) 1860-1866.

13. J. Jost, Riemannian geometry and geometric analysis, 2nd edn. (Universitext, Springer, New Mexico, 1998).

14. A. Lytchak, Geometric resolution of singular Riemannian foliations, Geom. Dedicata 149(1) (2010), 379-395.

15. A. Lytchak and G. Thorbergsson, Variationally complete actions on nonnegatively curved manifolds, Illinois J. Math. 51(2) (2007), 605-615.

16. A. Lytchak and G. Thorbergsson, Curvature explosion in quotients and applications, J. Differ. Geom. 85(1) (2010), 117-139.

17. I. Moerdijk and J. Mrčun, Introduction to foliations and lie groupoids, Cambridge Studies in Advanced Mathematics, vol. 91 (Cambridge University Press, Cambridge, UK, 2003).

18. P. Molino, Riemannian foliations, Progress in Mathematics vol. 73 (Birkhäuser, Boston, 1988).

19. C.-L. Terng and G. Thorbergsson, Submanifold geometry in symmetric spaces, J. Differ. Geom. 42(3) (1995), 665-718.

20. G. Thorbergsson, A survey on isoparametric hypersurfaces and their generalizations, in Handbook of differential geometry, vol. 1 (Elsevier Science B.V., Netherlands, 2000). 